\title{
Retrieving the Ultrasmall-Angle X-Ray Scattering Signal with Polychromatic Radiation in Speckle-Tracking and Beam-Tracking Phase-Contrast Imaging
}

\author{
Fabio A. Vittoria, ${ }^{*}$ Marco Endrizzi, and Alessandro Olivo \\ Department of Medical Physics and Biomedical Engineering, University College London, \\ Malet Place, Gower Street, London WCIE 6BT, United Kingdom \\ (Received 29 November 2016; revised manuscript received 10 February 2017; published 27 March 2017)

\begin{abstract}
We present an experimental comparison between two x-ray phase-contrast imaging techniques currently under development, speckle tracking and beam tracking. The comparison is centered on the absorption and ultrasmall-angle scattering signals retrieved with polychromatic radiation from homogeneous and inhomogeneous samples of different thicknesses. Our analysis shows that the ultrasmall-angle scattering signal retrieved with speckle tracking does not increase linearly with the thickness for the inhomogeneous sample, and is different from zero for the homogeneous sample. The results obtained from beam tracking, instead, are in good agreement with the expectation.
\end{abstract}

DOI: 10.1103/PhysRevApplied.7.034024

\section{INTRODUCTION}

$\mathrm{X}$-ray imaging is an important tool for the study and investigation of the internal structure of specimens in a large variety of fields. When $\mathrm{x}$ rays propagate through a material, they can be attenuated and/or refracted, depending on the chemical composition and density of the material's internal structures. The different attenuation properties of different materials constitute the only contrast mechanism in standard x-ray radiography. This is mainly because the angles at which $\mathrm{x}$ rays are refracted are particularly small, of the order of microradians or below. In x-ray phasecontrast imaging (XPCI), instead, refraction induced by the sample is translated into intensity modulation on the detector. This mechanism can result in a drastic increase in image contrast with respect to standard radiography, especially for low-absorbing materials. When the structures refracting $\mathrm{x}$ rays are smaller than the resolution of the imaging system, a third effect occurs, often referred to as ultrasmall-angle $\mathrm{x}$-ray scattering (USAXS) in the literature [1-3]. XPCI has been implemented with different approaches; some of the most commonly used methods include free-space propagation [4-6], analyzer-based imaging [7,8], grating interferometry [9-11], and edge illumination $[12,13]$. Recently, alternative XPCI methods have been proposed, based on the analysis of the distortions induced by the sample to a reference pattern created through an optical element. The reference pattern can be

\footnotetext{
*Corresponding author. fabio.vittoria.12@ucl.ac.uk

Published by the American Physical Society under the terms of the Creative Commons Attribution 4.0 International license. Further distribution of this work must maintain attribution to the author(s) and the published article's title, journal citation, and DOI.
}

created using the self-image of a phase grating [14], the near-field speckle pattern from a random object [15-17], or the projection image of a slit or an absorbing mask [18-21]. In particular, grating interferometry [2], edge illumination [3], speckle tracking [17], and beam tracking [21] have been successfully implemented with polychromatic laboratory sources, and dedicated algorithms have been developed to retrieve the absorption, refraction, and scattering signals of the sample. The beam-tracking method is an implementation, in the hard x-ray regime, of the Hartmann wave-front sensor, using laminar beamlets. The same concept can be found in the patent presented by Wilkins in 1995 [22], and in some later studies [23,24].

The possibility to extract the scattering signal is important in many applications [25-30], as it provides information on the micrometric structure of the sample that is not accessible through the other contrast mechanisms. The USAXS signal, in fact, originates from small structures in the sample that result in a variation of its projected index of refraction on a scale smaller than the effective resolution of the imaging system [31,32]. Moreover, it has been shown that, when monochromatic radiation is used, the scattering signal produced by microstructured samples increases linearly with the sample thickness, thus allowing its implementation in tomography for the reconstruction of the three-dimensional scattering map of a sample [33]. However, recent studies have shown that, when polychromatic radiation is used, a spurious scattering signal can be retrieved with grating interferometry, edge illumination, speckle tracking, and beam tracking, and that this spurious signal originates from the variation of the $\mathrm{x}$-ray mass attenuation coefficient of the sample with energy [21,34-36]. As a result, a scattering signal can be retrieved also from homogeneous objects, making the interpretations of scattering images obtained with the cited methods ambiguous in terms of sample microstructures. Strategies to correct for these artifacts are 
also presented in Refs. [21,36] for the beam-tracking and grating interferometry methods. Although no study has been presented yet, the results shown in Ref. [21] suggest the possibility that the scattering signal retrieved in edge illumination could be corrected in a similar way to the one proposed in Ref. [21].

The studies reported so far, however, only consider the signal retrieved from a homogeneous object. The aim of this work is to study how the scattering signal retrieved with polychromatic radiation in speckle tracking and beam tracking varies with the thickness not only for a homogeneous, but also for an inhomogeneous sample. Here "homogeneous" is used to indicate an object made of a single material with constant or slowly varying (compared to the resolution of the imaging system) projected thickness, while the term "inhomogeneous" refers to samples with internal structures smaller than the system resolution. The scattering signal should increase linearly with the sample thickness for inhomogeneous objects, and be equal to zero for homogeneous ones. In this study, we compare the beam-tracking and speckle-tracking methods due to the similarity and simplicity of the two approaches.

\section{METHODS}

The experimental setups used are shown in Fig. 1. The same source and detector are used for the two configurations. The source is a microfocus transmission tungsten target $\mathrm{x}$-ray tube, operating at $50 \mathrm{kVp}$ with a source size of about $3.5 \mu \mathrm{m}$. The detector is a CCD camera coupled through a fiber-optic plate to a Gadox scintillator with an effective pixel size equal to $4.54 \mu \mathrm{m}$ (Photonic Science). For the setup in Fig. 1(a), the speckle pattern is created through a piece of sandpaper with a mean grain size of $18.3 \mu \mathrm{m}$ (P1000 of FEPA $P$-grit classification). The sourceto-sandpaper, sandpaper-to-sample, and sample-to-detector

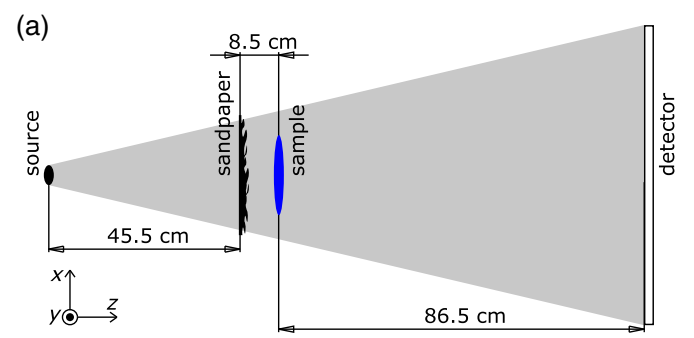

(b)

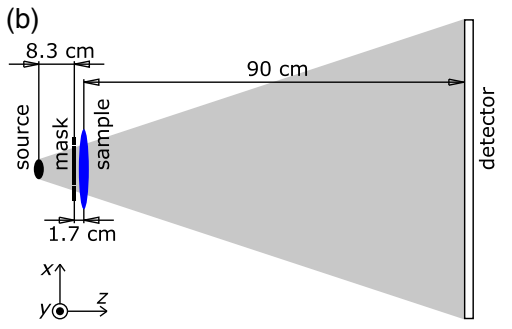

FIG. 1. Scheme of the experimental setups used for the speckletracking (a) and beam-tracking (b) methods. distances are 45.5, 8.5, and $86.5 \mathrm{~cm}$, respectively. For the beam-tracking configuration [Fig. 1(b)], the absorbing mask is made of gold on a silicon substrate with a period and aperture size of 20 and $3 \mu \mathrm{m}$, respectively. In this case, the source-to-mask, mask-to-sample, and sample-todetector distances are 8.3,1.7, and $90 \mathrm{~cm}$, respectively. It is important to note that the two methods are implemented with different geometries and magnifications. For the speckle-tracking method, a certain propagation distance between the source and the sandpaper is necessary in order to increase the degree of coherence of the radiation; however, an excessive increase of this distance would result in lower flux and higher image noise. Regarding the distance between the sandpaper and the detector, a larger propagation distance would contribute to an increase of the speckle visibility, but it would also increase the requirements in terms of coherence of the radiation illuminating the sandpaper. The speckle pattern measured by the detector, in fact, can be considered as the near-field propagation-based phase-contrast image of the sandpaper [15], and the coherence requirements for its formation can be analyzed in the framework previously developed for free-space propagation phase-contrast imaging [37]. The contrast transfer function (CTF) for the intensity pattern created through free-space propagation by a weak phase object illuminated by a monochromatic spherical wave can be approximated with the following expression [37]:

$$
\operatorname{CTF}(u)=\sin \left(\pi \lambda M z_{p} u^{2}\right),
$$

where $u$ is the frequency coordinate in Fourier space, $\lambda$ is the x-ray wavelength, $z_{p}$ is the sandpaper-to-detector distance, and $M$ is the geometrical magnification equal to the source-to-detector distance divided by the sandpaperto-detector distance. The CTF is an oscillating function equal to 0 for $u=0$, and whose first maximum is found at a frequency value of $\bar{u}=\left(2 \lambda M z_{p}\right)^{-1 / 2}$. For our setup, considering a mean energy of the radiation of $20 \mathrm{keV}$, we have $\bar{u}=5.2 \times 10^{4} \mathrm{~m}^{-1}$. The effect of finite source size (i.e., limited transversal coherence) and limited detector resolution can be described by a convolution of the intensity pattern with the projected source intensity distribution (PS) and the detector point spread function (PSF). Therefore, the CTF of an ideal system needs to be multiplied by the Fourier transforms of PS and PSF. The product of the last two functions can be interpreted as the modulation transfer function (MTF) of the system. For our particular experimental setup $\operatorname{MTF}(\bar{u}) 0.1$. While this implies that the phase-contrast signal is substantially reduced with respect to the ideal case, it also means that the low-frequency part of the signal (for $u \leq \bar{u}$ ), although modulated, is preserved. Under the above conditions of finite source size and limited detector resolution, the effect of polychromaticity (i.e., longitudinal coherence) is of secondary importance, and does not significantly affect the phase-contrast signal 
obtained in free-space propagation [37]. For the beamtracking method it is important to keep the different beamlets created by the mask, and measured by the detector separated. For the particular setup used, the main source of broadening of the beamlets is the detector point spread function. For this reason, and for the relatively small period of the sample mask, we decided to use a high magnification value. The PSF of the detector used, in fact, is characterized by a relatively small full width at half maximum of approximately $10 \mu \mathrm{m}$, but also by the presence of long "tails". We estimate the width along the $x$ direction over which the area of the PSF is $99 \%$ of the total to be of about $120 \mu \mathrm{m}$. If blurring due to source size and mask apertures is considered, this width becomes equal to $\approx 145 \mu \mathrm{m}$, while the period of the sample mask, magnified at the detector plane, is $\approx 240 \mu \mathrm{m}$. This ensures a good separation between the different beamlets. The two different geometries might affect differently the two methods in terms of sensitivity to the phase signal and resolution due to, for example, the different propagation distance and magnification. This, however, does not affect the main results of this study in terms of average absorption and ultrasmall-angle scattering signal retrieved from the different objects. The test sample used in the experiment is schematically represented in Fig. 2. The top part consists of different layers of a plastic material; each layer is $850 \mu \mathrm{m}$ thick and the number of layers increases from left to right. The bottom part consists of different layers of paper; each layer is made of three superimposed sheets, and the number of layers decreases from left to right. In the speckle-tracking configuration four images are acquired and averaged for each acquisition with an exposure time of $900 \mathrm{~s}$ per image, and four reference images without the sample are acquired and averaged with the same exposure time. Four different acquisitions are performed to cover the different areas of interest of the sample. In the beam-tracking configuration, each acquisition consists of an eight-step scan of the sample over one period of the mask magnified at the sample plane (dithering

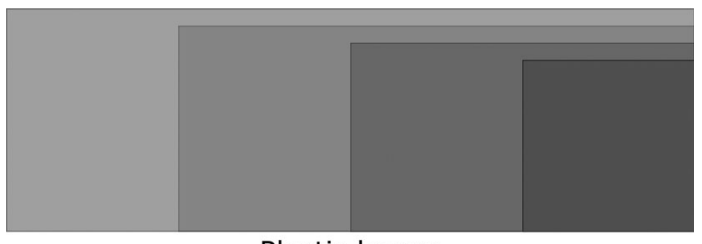

Plastic layers

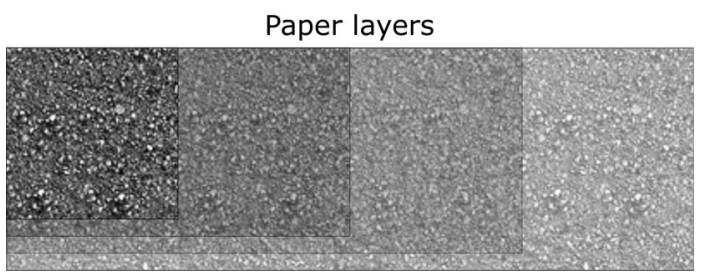

FIG. 2. Schematic representation of the test sample used. procedure), along the direction orthogonal to the mask apertures, with 900-s exposure time for each step. Also in this case four reference images are acquired and averaged without the sample with 900-s exposure time per image. Eight acquisitions are needed to cover the areas of interest of the sample. For each acquisition the integrated air kerma at the sample plane is approximately 0.4 and $0.7 \mathrm{~Gy}$ for speckle tracking and beam tracking, respectively. Four images, with 900-s exposure time per image, are acquired with the x-ray source off, and averaged to measure the detector dark current. Four images, with 900-s exposure time per image, are acquired with the x-ray source on and with no optical element and/or sample in the beam, and averaged to form a "flat-field" image. All experimental images are corrected by subtracting the detector dark current and dividing by the flat-field image minus the detector dark current.

An example of the speckle pattern obtained with the described setup is shown in Fig. 3(a), and a profile extracted from the image is shown in Fig. 3(b). The Fourier power spectrum of the speckle pattern shows a maximum at a frequency value equal to approximately $1 / 26$ pixel $^{-1}$. This value can be considered as the inverse of the average period of the speckle pattern and will be used later to determine the window size used in the analysis of the speckle images. When a sample is introduced in the beam, the speckle pattern is locally distorted due to absorption, refraction, and scattering. This can be mathematically described by the following equations [17]:

$$
\begin{aligned}
I_{r}(x, y) & =I_{0}+\Delta I_{r}(x, y) ; \\
I(x, y) & =T\left[I_{0}+D \Delta I_{r}\left(x-\delta_{x}, y-\delta_{y}\right)\right] .
\end{aligned}
$$

$I_{r}$ is the reference intensity, measured without the sample, and is described as the sum of a constant term $I_{0}$ and an oscillating term $\Delta I_{r}$. I is the intensity measured when the sample is in the beam, and it is related to $I_{r}$ through the constant parameters $T, D, \delta_{x}, \delta_{y} . T$ describes the absorption of the sample, and reduces the mean value of the intensity
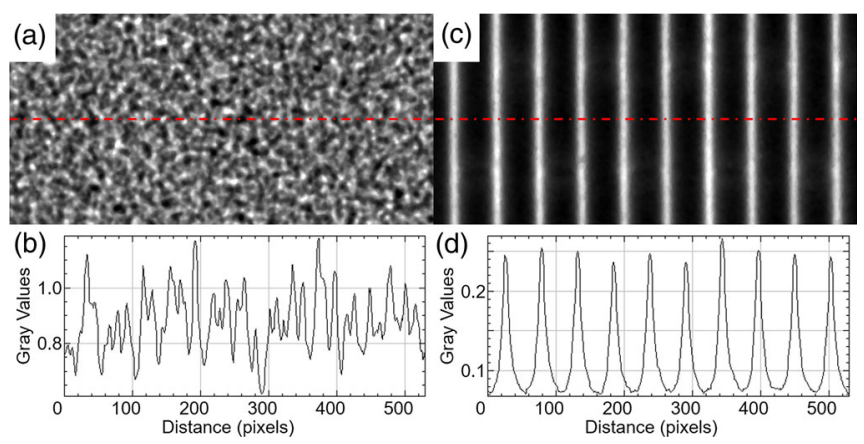

FIG. 3. Example of the intensity pattern measured in the speckle-tracking (a) and beam-tracking (c) configurations. Extracted profiles are shown in (b) and (d). 
pattern; $D$ describes the scattering of the sample, and reduces the visibility of the intensity pattern; $\delta_{x}$ and $\delta_{y}$ describe the refraction induced by the sample along the horizontal and vertical direction, respectively, and change the lateral position of the speckles. $T, D, \delta_{x}, \delta_{y}$ are retrieved using the minimization procedure described in Ref. [17]. For each point $\left(x_{0}, y_{0}\right)$ of the measured intensity pattern the following cost function is calculated:

$$
\begin{aligned}
f\left(x_{0}, y_{0} ; T, D, \delta_{x}, \delta_{y}\right) \\
=\int\left\{T\left[I_{0}+D \Delta I_{r}\left(x-\delta_{x}, y-\delta_{y}\right)\right]-I(x, y)\right\}^{2} \\
\quad \times w\left(x-x_{0}, y-y_{0}\right) d x d y,
\end{aligned}
$$

and minimized with respect to $T, D, \delta_{x}, \delta_{y} . w\left(x-x_{0}\right.$, $\left.y-y_{0}\right)$ is a smooth circular window function centered in $\left(x_{0}, y_{0}\right)$, obtained from the convolution of two circular top-hat functions with a radii of 26 and 13 pixels. The resulting full width at half maximum of the window function is approximately 52 pixels, equal to twice the average period of the speckle pattern.

Figure 3(c) shows the image of the mask obtained with the beam-tracking setup, and an extracted profile is shown in Fig. 3(d). For the case considered here, each reference beam has been described as the sum of a constant term and two Gaussian functions [21]:

$$
\begin{aligned}
I_{r}(x)= & A_{0}+\frac{A_{1}}{\left[2 \pi \sigma_{1}^{2}\right]^{1 / 2}} \exp \left[-\frac{\left(x-\mu_{1}\right)^{2}}{2 \sigma_{1}^{2}}\right] \\
& +\frac{A_{2}}{\left[2 \pi \sigma_{2}^{2}\right]^{1 / 2}} \exp \left[-\frac{\left(x-\mu_{2}\right)^{2}}{2 \sigma_{2}^{2}}\right] .
\end{aligned}
$$

The constant term describes the radiation transmitted through the gold layer of the mask. For an ideal system, this term should be as close as possible to zero; for this experiment, however, we use a mask with relatively high transmission through the gold layer to test the retrieval results in nonideal conditions. The number of Gaussian terms is arbitrary and chosen to best reproduce the experimentally observed beamlet shape. For this specific case each beamlet presents relatively long tails, and while the first Gaussian describes the central part of the beamlet, the second one is used for the tails. When the sample is introduced in the beam, its intensity profile can be described as [21]:

$$
\begin{aligned}
I(x)= & B_{0}+T \frac{A_{1}}{\left[2 \pi\left(\sigma_{1}^{2}+\sigma_{D}^{2}\right)\right]^{1 / 2}} \exp \left[-\frac{\left(x-\mu_{1}-\delta_{x}\right)^{2}}{2\left(\sigma_{1}^{2}+\sigma_{D}^{2}\right)}\right] \\
& +T \frac{A_{2}}{\left[2 \pi\left(\sigma_{2}^{2}+\sigma_{D}^{2}\right)\right]^{1 / 2}} \exp \left[-\frac{\left(x-\mu_{2}-\delta_{x}\right)^{2}}{2\left(\sigma_{2}^{2}+\sigma_{D}^{2}\right)}\right],
\end{aligned}
$$

where $T$ describes absorption, $\delta_{x}$ refraction in the direction orthogonal to the mask apertures, and $\sigma_{D}$ scattering. Note that the radiation transmitted through the gold layer $\left[A_{0}\right.$ in Eq. (5)] is partially absorbed by the sample [becoming $B_{0}$, see Eq. (6)]; however, since in general, the spectrum of the radiation transmitted through the gold layer is different from the one traveling through the apertures, $B_{0} / A_{0} \neq T$ [21]. $T, \delta_{x}$, and $\sigma_{D}$ are retrieved by a multi-Gaussian fitting of the intensity profiles measured with and without the sample [21]. It is important to note that all the quantities retrieved with the two methods depend on the spectrum impinging on the sample and that this is different in the two cases. For the beam-tracking case, theoretical expressions for the dependency of $T, \delta_{x}$, and $\sigma_{D}$ on the source spectrum, detector energy response, and mask composition and thickness can be found in Ref. [21]. For the beam-tracking configuration, the sampling step is different in the two directions, as it is equal to the step used in the dithering procedure $(\approx 3 \mu \mathrm{m})$ in the horizontal direction $(x)$, and to the demagnified pixel size $(\approx 0.45 \mu \mathrm{m})$ in the vertical direction $(y)$. Experimental data are binned by a factor of 6 in the vertical $(y)$ direction to reduce noise and obtain a similar sampling step in the reconstructed signals in the two directions. The binning procedure affects only the final resolution of the images and the level of noise, without changing the nature of the retrieved signals. While absorption and refraction are described through the same quantities in the two models [ $T$ and $\delta_{x}$ in Eqs. (3) and (6)], the contribution due to scattering appears in different forms: as a loss of visibility in Eq. (3), and as an increase in the beam width in Eq. (6). These two descriptions are equivalent when the effect of scattering can be described as a convolution of the reference pattern with a Gaussian scattering function, and if the reference pattern is a periodic sinusoidal function. In this case it can be shown that

$$
\sigma_{D}^{2} \propto-\ln D
$$

where $D$ is the loss of visibility of the sinusoidal reference function, and $\sigma_{D}$ is the standard deviation of the Gaussian scattering function. For this reason, we will compare the signal $-\ln D$, obtained from speckle tracking, with $\sigma_{D}^{2}$, obtained from beam tracking. Finally, it is important to note that, due to the one-dimensional structure of the pattern created through the absorbing mask [Fig. 3(c)], beam tracking is sensitive only to the refraction signal $\delta_{x}$ along the $x$ direction, while with speckle tracking it is possible to retrieve both $\delta_{x}$ and $\delta_{y}$. Beam tracking could be extended to two-directional sensitivity by using a mask featuring an array of holes rather than long slits.

\section{RESULTS}

Figure 4 shows the signals retrieved with the speckletracking configuration. The absorption signal in Fig. 4(a) is calculated as $-\ln T$, while the scattering signal in Fig. 4(b) 


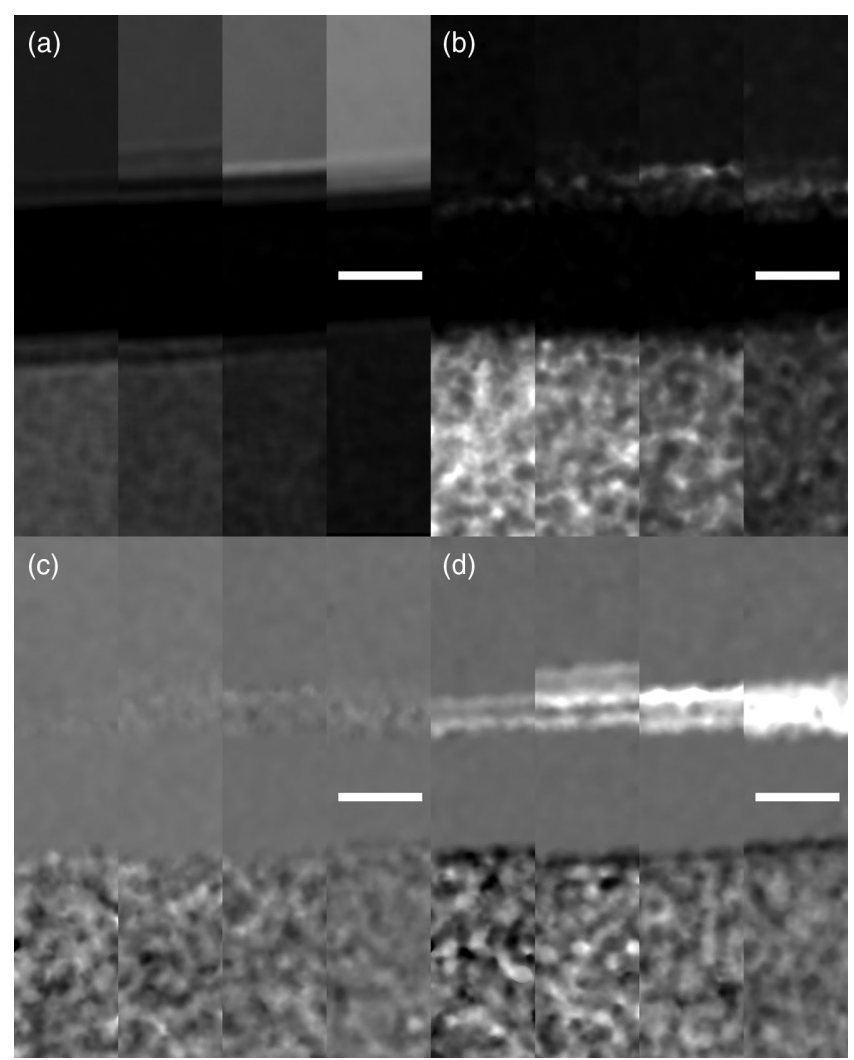

FIG. 4. Signals retrieved in the speckle-tracking configuration: absorption (a), scattering (b), and refraction along the horizontal (c) and vertical (d) direction. The display ranges are $[0,0.5]$ in (a), $[0,1.5]$ in (b), $[-10,10] \mu \mathrm{rad}$ in (c) and (d). The scale bar corresponds to $500 \mu \mathrm{m}$.

is calculated as $-\ln D$. The refraction signals, retrieved as a variation of the speckles position at the detector, are displayed as refraction angles $\delta_{x} / z_{s d}$ and $\delta_{y} / z_{s d}$ in Figs. 4(c) and 4(d), respectively, where $z_{s d}$ is the sampleto-detector distance. Similarly, Fig. 5 shows the signals retrieved from the beam-tracking configuration. In this case, the scattering signal is calculated as $\sigma_{D}^{2} / z_{s d}^{2}$. It can be seen how the images in Fig. 5 presents a higher resolution than the ones in Fig. 4; this, however, is not an intrinsic advantage of the beam-tracking technique over speckle tracking, but only a result of the particular geometry and acquisition scheme used for this experiment. Speckle tracking, for example, can be implemented in a "speckle-scanning" configuration [38] which allows higher resolution, and requires different images acquired while performing a raster scan of the scattering element. Additionally, the higher resolution of the beam-tracking configuration comes at the cost of reduced radiation efficiency due to reduced transmission through the mask compared to the sandpaper. Image resolution is not important for the purpose of our study, as we are interested in the area contrast of the absorption and scattering signals for different samples.

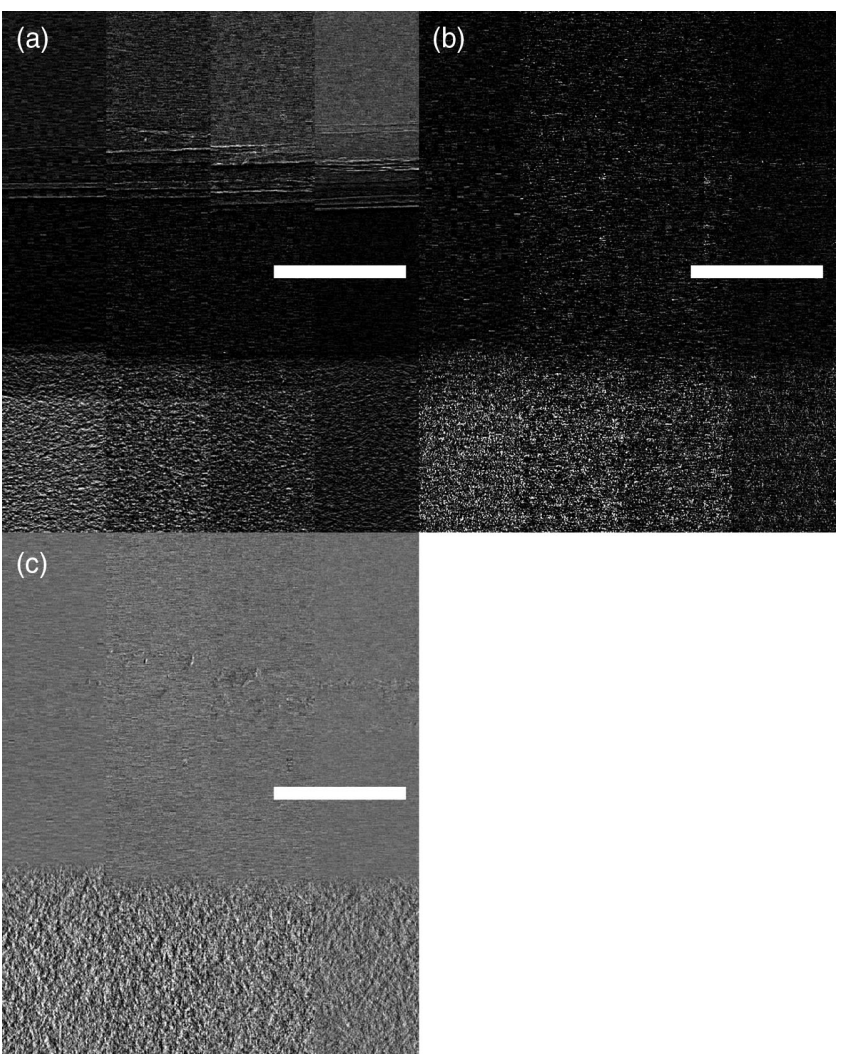

FIG. 5. Signals retrieved in the beam-tracking configuration: absorption (a), scattering (b), and refraction along the horizontal (c) direction. The display ranges are $[0,0.5]$ in (a), $[0,250] \mu \mathrm{rad}^{2}$ in (b), $[-10,10] \mu \mathrm{rad}$ in (c). The scale bar corresponds to $500 \mu \mathrm{m}$.

For each material and thickness the average values of absorption and scattering are calculated over an area of $30 \times 5$ pixels in the speckle-tracking case, and $80 \times 20$ pixels in the beam-tracking case. In the speckle-tracking case, the window used for the retrieval introduces a strong correlation between the retrieved values in adjacent pixels; for this reason the selected $30 \times 5$ pixels are not adjacent, but at a distance of 52 pixels from one another. The results are shown in Figs. 6(a)-6(d), together with a linear fit of the experimental values. The retrieval procedures used for the two methods assume simple models to describe how the sample distorts the reference intensity pattern [Eqs. (3) and (6)]. From Eq. (3) it is possible to write

$$
\Delta I(x, y)=I(x, y)-\bar{I}=T D \Delta I_{r}\left(x-\delta_{x}, y-\delta_{y}\right),
$$

where $\bar{I}$ is the average value of $I(x, y)$. Let us introduce the normalized cross-correlation between $\Delta I$ and $\Delta I_{r}$ :

$\rho\left(x_{0}, y_{0}\right)=\frac{\int \Delta I(x, y) \Delta I_{r}\left(x+x_{0}, y+y_{0}\right) d x d y}{\left[\int \Delta I^{2}(x, y) d x d y \int \Delta I_{r}^{2}(x, y) d x d y\right]^{1 / 2}}$.

By assuming that Eq. (3) accurately describes the imageformation process, one obtains 


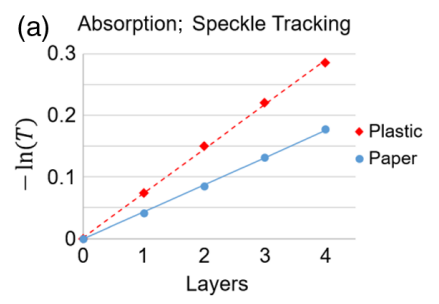

(c) Scattering; Speckle Tracking

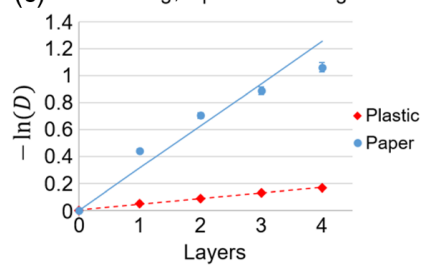

(e) Cross-Correlation; Speckle Tracking
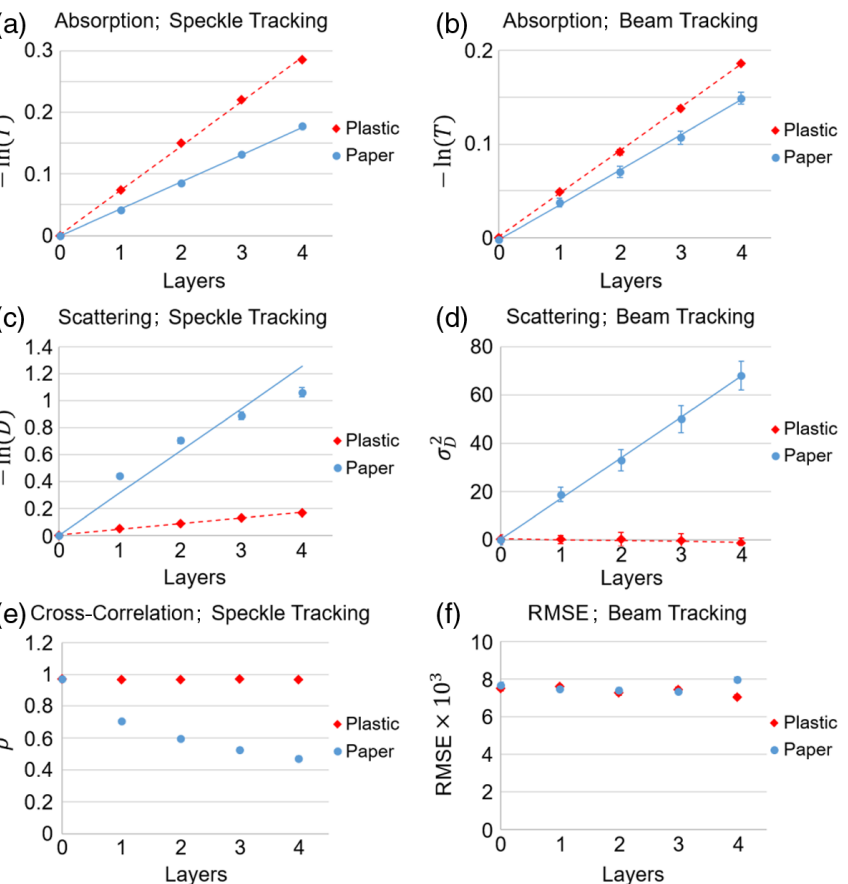

(d) Scattering; Beam Tracking
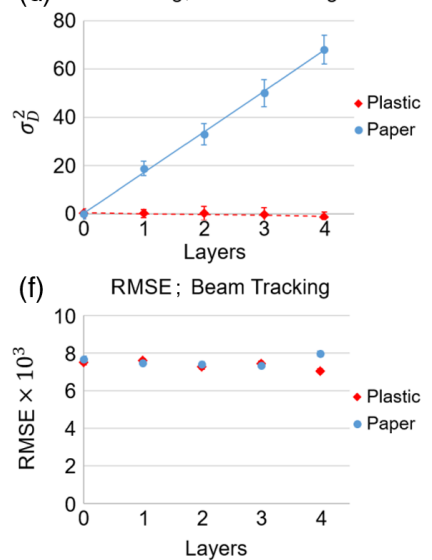

FIG. 6. Average retrieved values of absorption for speckle tracking (a) and beam tracking (b), and of scattering for speckle tracking (c) and beam tracking (d). Cross-correlation between the speckle pattern with and without the sample (e). Root-meansquare error of the Gaussian fit used for the beam-tracking retrieval (f). In all graphs, the experimental values referring to the plastic samples are indicated with red diamonds, the values for the paper samples are indicated with blue circles. In (a)-(d) a linear fit of the experimental values is shown with a red dashed line for the plastic samples, and with a blue solid line for the paper sample. Error bars indicate the 95\% confidence interval; where not visible, the confidence interval is smaller than the marker size.

$$
\rho\left(-\delta_{x},-\delta_{y}\right)=1 .
$$

We calculate $\rho\left(-\delta_{x},-\delta_{y}\right)$ over the same window used for the retrieval around each pixel in the $30 \times 5$ area mentioned before. The average values of $\rho\left(-\delta_{x},-\delta_{y}\right)$ obtained for the different materials and thicknesses are shown in Fig. 6(e). Equation (6) assumes that scattering can be described as a convolution of the reference beam with a Gaussian scattering function. To test if the accuracy of this model is influenced by the sample material or thickness, we calculate the root-mean-square error between the experimental beam profiles and the Gaussian fit over the $(80 \times 20)$-pixel area mentioned before. The obtained mean values are shown in Fig. 6(f).

\section{DISCUSSION}

The retrieved absorption signal is expected to increase linearly with the material thickness (neglecting the effect of beam hardening caused by the sample). This behavior is observed for both methods with both materials (plastic and paper). One important difference, however, is the absolute values of the retrieved absorption signal: the beam-tracking method provides lower absorption values than speckle tracking. This difference is likely to be caused by the different spectra of the radiation impinging on the sample in the two cases: the mask used in the beam-tracking setup has a 500- $\mu$ m-thick silicon substrate, which acts as a filter, and increases the mean energy of the radiation on the sample.

The scattering signal shows significant differences between the two methods. For the plastic sample, a nonzero scattering signal is retrieved with speckle tracking, which increases linearly with the thickness of the material. This confirms the simulation results presented in a previous study [36]. Beam tracking does not appear to be affected by this problem, and the retrieved signal is approximately zero for the range of investigated thicknesses. The scattering signal retrieved from the inhomogeneous sample (paper) increases linearly with the material thickness, as expected, for the beam-tracking configuration, while it increases sublinearly in the speckle-tracking case. While it is not trivial to understand the cause of this behavior, an explanation can be found by analyzing how the cross-correlation between the speckle pattern measured with and without the sample changes for the different samples and material thicknesses [Fig. 6(e)]. The cross-correlation is approximately constant and near unity for the plastic sample, indicating that the "structure" of the speckle pattern is not altered by the plastic layers. This also confirms that the properties of the speckle pattern are not significantly modified by the beam hardening introduced by the sample to the x-ray beam [15]. A different result can be observed for the paper sample, for which the correlation decreases when the sample thickness increases. This result indicates that not only does scattering reduce the visibility of the speckle pattern, but it also changes its structure. A similar comparison has been performed for the beam-tracking case [Fig. 6(f)] using the root-mean-square error of the fit procedure. In this case, no clear trend can be observed.

\section{CONCLUSION}

We compare how the absorption and scattering signals retrieved in speckle-tracking and beam-tracking XPCI depend on the sample internal microstructure and thickness when polychromatic radiation is used. Both signals are expected to increase linearly with the material thickness, and no scattering should be retrieved from homogeneous materials. The linearity of the signal with the sample thickness is of particular importance for a tomographic application with laboratory sources of the two techniques. We find that the scattering signal retrieved in speckle tracking is different from zero and increases linearly with the thickness for a homogeneous plastic sample, while it increases sublinearly for an inhomogeneous sample made of paper. While the nonzero scattering signal retrieved from 
homogeneous objects is expected from simulations, it is not related to microstructure in the sample. Further studies should be conducted to understand the nature of these artifacts and the possibility to correct them. The scattering signal retrieved in beam tracking follows the expected trend for the range of thicknesses we have investigated. Finally, the absorption signal retrieved with both modalities follows the expected linear trend.

\section{ACKNOWLEDGMENTS}

This work is funded by the EPSRC (Grants No. EP/ M507970/1 and No. EP/I021884/1). M. E. is supported by the Royal Academy of Engineering under the RAEng Research Fellowships scheme. The authors would like to thank Pierre Thibault and Irene Zanette for comments that greatly improved the manuscript.

[1] L. Rigon, F. Arfelli, and R. Menk, Generalized diffraction enhanced imaging to retrieve absorption, refraction and scattering effects, J. Phys. D 40, 3077 (2007).

[2] F. Pfeiffer, M. Bech, O. Bunk, P. Kraft, E. F. Eikenberry, C. Brönnimann, C. Grünzweig, and C. David, Hard$\mathrm{X}$-ray dark-field imaging using a grating interferometer, Nat. Mater. 7, 134 (2008).

[3] M. Endrizzi, P. C. Diemoz, T. P. Millard, J. Louise Jones, R. D. Speller, I. K. Robinson, and A. Olivo, Hard X-ray dark-field imaging with incoherent sample illumination, Appl. Phys. Lett. 104, 024106 (2014).

[4] A. Snigirev, I. Snigireva, V. Kohn, S. Kuznetsov, and I. Schelokov, On the possibilities of $\mathrm{x}$-ray phase contrast microimaging by coherent high-energy synchrotron radiation, Rev. Sci. Instrum. 66, 5486 (1995).

[5] S. W. Wilkins, T. E. Gureyev, D. Gao, A. Pogany, and A. W. Stevenson, Phase-contrast imaging using polychromatic hard X-rays, Nature (London) 384, 335 (1996).

[6] J. Moosmann, A. Ershov, V. Weinhardt, T. Baumbach, M. S. Prasad, C. LaBonne, X. Xiao, J. Kashef, and R. Hofmann, Time-lapse $\mathrm{x}$-ray phase-contrast microtomography for in vivo imaging and analysis of morphogenesis, Nat. Protoc. 9 , 294 (2014).

[7] E. Förster, K. Goetz, and P. Zaumseil, Double crystal diffractometry for the characterization of targets for laser fusion experiments, Krist. Tech. 15, 937 (1980).

[8] T. J. Davis, D. Gao, T. E. Gureyev, A. W. Stevenson, and S. W. Wilkins, Phase-contrast imaging of weakly absorbing materials using hard x-rays, Nature (London) 373, 595 (1995).

[9] C. David, B. Nohammer, H. H. Solak, and E. Ziegler, Differential x-ray phase contrast imaging using a shearing interferometer, Appl. Phys. Lett. 81, 3287 (2002).

[10] A. Momose, S. Kawamoto, I. Koyama, Y. Hamaishi, K. Takai, and Y. Suzuki, Demonstration of X-ray talbot interferometry, Jpn. J. Appl. Phys. 42, L866 (2003).

[11] F. Pfeiffer, T. Weitkamp, O. Bunk, and C. David, Phase retrieval and differential phase-contrast imaging with lowbrilliance x-ray sources, Nat. Phys. 2, 258 (2006).
[12] A. Olivo, F. Arfelli, G. Cantatore, R. Longo, R. H. Menk, S. Pani, M. Prest, P. Poropat, L. Rigon, G. Tromba, E. Vallazza, and E. Castelli, An innovative digital imaging setup allowing a low-dose approach to phase contrast applications in the medical field, Med. Phys. 28, 1610 (2001).

[13] A. Olivo and R. Speller, A coded-aperture approach allowing x-ray phase contrast imaging with conventional sources, Appl. Phys. Lett. 91, 074106 (2007).

[14] K. S. Morgan, P. Modregger, S. C. Irvine, S. Rutishauser, V. A. Guzenko, M. Stampanoni, and C. David, A sensitive $\mathrm{X}$-ray phase contrast technique for rapid imaging using a single phase grid analyzer, Opt. Lett. 38, 4605 (2013).

[15] K. S. Morgan, D. M. Paganin, and K. K. W. Siu, X-ray phase imaging with a paper analyzer, Appl. Phys. Lett. 100, 124102 (2012).

[16] S. Bérujon, E. Ziegler, R. Cerbino, and L. Peverini, TwoDimensional X-Ray Beam Phase Sensing, Phys. Rev. Lett. 108, 158102 (2012).

[17] I. Zanette, T. Zhou, A. Burvall, U. Lundström, D. H. Larsson, M. Zdora, P. Thibault, F. Pfeiffer, and H. M. Hertz, Speckle-Based X-Ray Phase-Contrast and Dark-Field Imaging with a Laboratory Source, Phys. Rev. Lett. 112, 253903 (2014).

[18] F. Krejci, J. Jakubek, and M. Kroupa, Single grating method for low dose 1-D and 2-D phase contrast x-ray imaging, J. Instrum. 6, C01073 (2011).

[19] H. H. Wen, E. E. Bennett, R. Kopace, A. F. Stein, and V. Pai, Single-shot X-ray differential phase-contrast and diffraction imaging using two-dimensional transmission gratings, Opt. Lett. 35, 1932 (2010).

[20] F. A. Vittoria, M. Endrizzi, P. C. Diemoz, U. H. Wagner, C. Rau, I. K. Robinson, and A. Olivo, Virtual edge illumination and one dimensional beam tracking for absorption, refraction, and scattering retrieval, Appl. Phys. Lett. 104, 134102 (2014).

[21] F. A. Vittoria, G. K. N. Kallon, D. Basta, P. C. Diemoz, I. K. Robinson, A. Olivo, and M. Endrizzi, Beam tracking approach for single-shot retrieval of absorption, refraction and dark-field signals with laboratory x-ray sources, Appl. Phys. Lett. 106, 224102 (2015).

[22] S. W. Wilkins, Improved x-ray optics, especially for phase contrast imaging, WIPO Patent No. WO 1995005725 A1 (23 February 1995).

[23] K. L. Baker, J. Brase, M. Kartz, S. S. Olivier, B. Sawvel, and J. Tucker, Electron density characterization by use of a broadband $\mathrm{x}$-ray-compatible wave-front sensor, Opt. Lett. 28, 149 (2003).

[24] S. C. Mayo and B. Sexton, Refractive microlens array for wave-front analysis in the medium to hard $\mathrm{x}$-ray range, Opt. Lett. 29, 866 (2004).

[25] A. Yaroshenko, F. G. Meinel, M. Bech, A. Tapfer, A. Velroyen, S. Schleede, S. Auweter, A. Bohla, A. Ö. Yildirim, K. Nikolaou, F. Bamberg, O. Eickelberg, M. F. Reiser, and F. Pfeiffer, Pulmonary emphysema diagnosis with a preclinical small-animal $\mathrm{x}$-ray dark-field scattercontrast scanner, Radiology 269, 427 (2013).

[26] Z. Wang, N. Hauser, G. Singer, M. Trippel, R. A. KubikHuch, C. W. Schneider, and M. Stampanoni, Non-invasive classification of microcalcifications with phase-contrast x-ray mammography, Nat. Commun. 5, 3797 (2014). 
[27] P. Modregger, M. Kagias, S. Peter, M. Abis, V. A. Guzenko, C. David, and M. Stampanoni, Multiple Scattering Tomography, Phys. Rev. Lett. 113, 020801 (2014).

[28] M. Endrizzi, B. I. S. Murat, P. Fromme, and A. Olivo, Edgeillumination $\mathrm{X}$-ray dark-field imaging for visualising defects in composite structures, Compos. Struct. 134, 895 (2015).

[29] T. P. Millard, M. Endrizzi, N. Everdell, L. Rigon, F. Arfelli, R. H. Menk, E. Stride, and A. Olivo, Evaluation of microbubble contrast agents for dynamic imaging with $\mathrm{x}$-ray phase contrast, Sci. Rep. 5, 12509 (2015).

[30] P. Modregger, T. P. Cremona, C. Benarafa, J. C. Schittny, A. Olivo, and M. Endrizzi, Small angle X-ray scattering with edge-illumination, Sci. Rep. 6, 30940 (2016).

[31] W. Yashiro, Y. Terui, K. Kawabata, and A. Momose, On the origin of visibility contrast in X-ray talbot interferometry, Opt. Express 18, 16890 (2010).

[32] S. K. Lynch, V. Pai, J. Auxier, A. F. Stein, E. E. Bennett, C. K. Kemble, X. Xiao, W. Lee, N. Y. Morgan, and H. H. Wen, Interpretation of dark-field contrast and particle-size selectivity in grating interferometers, Appl. Opt. 50, 4310 (2011).
[33] L. Rigon, A. Astolfo, F. Arfelli, and R. Menk, Generalized diffraction enhanced imaging: Application to tomography, European Journal of Radiology 68S, S3 (2008).

[34] M. Endrizzi and A. Olivo, Absorption, refraction and scattering retrieval with an edge-illumination-based imaging setup, J. Phys. D 47, 505102 (2014).

[35] W. Yashiro, P. Vagovič, and A. Momose, Effect of beam hardening on a visibility-contrast image obtained by x-ray grating interferometry, Opt. Express 23, 23462 (2015).

[36] M. Zdora, P. Thibault, F. Pfeiffer, and I. Zanette, Simulations of X-ray speckle-based dark-field and phase-contrast imaging with a polychromatic beam, J. Appl. Phys. 118, 113105 (2015).

[37] A. Pogany, D. Gao, and S. W. Wilkins, Contrast and resolution in imaging with a microfocus x-ray source, Rev. Sci. Instrum. 68, 2774 (1997).

[38] S. Berujon, H. Wang, and K. Sawhney, X-ray multimodal imaging using a random-phase object, Phys. Rev. A 86, 063813 (2012). 